
allemande

45-2 | 2013

Images et discours de la nation

\title{
La foi des « Malgré Nous » (1942-1945)
}

À propos de : Lettres de Malgré-Nous. Témoignages d'incorporés de force alsaciens (Société d'histoire de la Poste et de France Télécom en Alsace, Strasbourg, La Nuée Bleue, 2012)

\section{Matthieu Arnold}

\section{(2penEdition}

\section{Journals}

Édition électronique

URL : https://journals.openedition.org/allemagne/1761

DOI : 10.4000/allemagne. 1761

ISSN : 2605-7913

Éditeur

Société d'études allemandes

\section{Édition imprimée}

Date de publication : 30 décembre 2013

Pagination : 449-456

ISSN : 0035-0974

\section{Référence électronique}

Matthieu Arnold, «La foi des « Malgré Nous » (1942-1945) », Revue d'Allemagne et des pays de langue allemande [En ligne], 45-2 | 2013, mis en ligne le 29 juillet 2019, consulté le 19 mai 2021. URL : http:// journals.openedition.org/allemagne/1761 ; DOI : https://doi.org/10.4000/allemagne.1761 


\section{La foi des «Malgré Nous » (1942-1945)*}

\section{- Matthieu Arnold**}

Nombreuses sont les études qui ont été consacrées à la foi et à la guerre durant la Première Guerre mondiale ${ }^{(1)}$. La Seconde Guerre mondiale, qui, il est vrai, n'a pas donné lieu aux mêmes excès du « Gott mit uns » que le conflit un quart de siècle auparavant ${ }^{(2)}$, n'a guère suscité de travaux sur cette question ${ }^{(3)}$. À partir d'un volume de correspondance récent, édité par un collectif d'historiens, nous aimerions montrer toute la légitimité d'une telle recherche: même si les questions d'intendance (la nourriture, les conditions de vie, le souci pour la famille... et pour la ferme pour les agriculteurs) revêtent souvent une place prépondérante dans ces lettres, les références à Dieu et à la religion sont loin d'en être absentes.

Le 25 août 1942, le Gauleiter Robert Wagner décrétait l'incorporation de force des Alsaciens et des Mosellans. Ces dernières années, une abondante littérature

* À propos de: Société d'histoire de la Poste et de France Télécom en Alsace, Lettres de Malgré-Nous. Témoignages d'incorporés de force alsaciens, Strasbourg, La Nuée Bleue, 2012, 271 p., ISBN 978-27165-0875-5, € 25.

** Professeur à la Faculté de Théologie protestante de l'Université de Strasbourg, GRENEP (EA 4378).

1 Voir les références dans Matthieu ARNOLD, «La chaire au service de la patrie: prédications protestantes françaises et allemandes durant la Première Guerre mondiale ", Revue d'Allemagne et des pays de langue allemande, 36/2 (2004), p. 135-154; ID., "Violence et image de Dieu dans les prédications protestantes de 1914-1918 ", in: Matthieu Arnold, Jean-Marc Prieur (éd.), Dieu est-il violent? La violence dans les représentations de Dieu, Strasbourg, Presses Universitaires, 2005, p. 69-88.

2 «Von Begeisterung konnte jedoch bei ihnen [= bei den evangelischen und katholischen Militärpfarrern] genau so wenig die Rede sein wie bei den Soldaten oder der Bevölkerung. Wenn auch hier und da der "Geist von 1914" beschworen wurde - die Erinnerungen an die Schrecken jener Jahre des "Weltkrieges” waren noch zu frisch, als daß ein neuerlicher Waffengang Grund zum Jubel gewesen wäre. » (Dieter BeEse, « Kirche im Krieg. Evangelische Wehrmachtpfarrer und die Kriegführung der deutschen Wehrmacht », in: Rolf-Dieter Müller, Hans-Erich Volkmann [éd.], Die Wehrmacht. Mythos und Realität, Munich, Oldenbourg, 1999, p. 486-502, ici p. 487.)

3 Outre les études auxquelles nous nous référons ci-après, voir notamment: Klaus Latzel, Vom Sterben im Krieg. Wandlungen in der Einstellung zum Tod vom Siebenjährigen Krieg bis zum II. Weltkrieg, Warendorf, Fahlbusch \& Co., 1988. 
- témoignages et études - a traité des Malgré Nous, au point que l'on peut faire de ce thème un des sous-genres de la littérature "alsatique ». Toutefois, le volume paru à l'automne de 2012 aux éditions de La Nuée Bleue constitue le premier recueil de lettres de Malgré Nous; ces lettres sont non seulement transcrites et le plus souvent traduites (la langue obligatoire pour la correspondance était l'allemand), mais aussi données en photographies, avec des reproductions d'une qualité telle qu'il est aisé de les lire et de contrôler les traductions.

Les éditeurs (vingt lecteurs-traducteurs, six historiens) ont sélectionné, dans près de 5000 lettres collectées par la Société d'Histoire de la Poste et de France Télécom en Alsace, des textes poignants, souvent accompagnés de photographies, et émanant « des incorporés de force présents dans les différentes armes et sur les différents fronts de la Wehrmacht entre 1943 et 1945, sans oublier ceux et celles qui ont dû servir sous le régime du [...] Reichsarbeitsdienst [...] ni les "Malgré-Elles", parfois versées dans les forces d'appui à la Wehrmacht » (p. 11). Le front Est est le plus représenté, la majorité des Alsaciens-Mosellans y ayant été envoyés; ils écrivent de Pologne, de Russie, de Lettonie $^{(4)}$... Pour autant, servir sur le front Ouest n'était pas un gage de survie: ainsi, le jeune René Sorgius, né le 16 février 1926 à Rosheim, fut tué le 7 juillet 1944 à Angoville-sur-Ay, durant les durs combats dans le bocage normand (p. 173). À côté de soldats de la Wehrmacht, les plus nombreux, on trouve quelques incorporés dans la Luftwaffe ou dans la Kriegsmarine ${ }^{(5)}$. Les documents sont présentés dans un ordre chronologique.

Les Malgré Nous de pas moins de 21 classes d'âges ont servi dans la Wehrmacht: les plus âgés (1908) ont été incorporés en avril 1944, les plus jeunes (1929) en janvier 1945. Ainsi, André Schwein, auteur d'une lettre rédigée le $1^{\text {er }}$ janvier 1945, et qui eut la chance de rentrer en Alsace le 10 juin 1945, est-il né à Grussenheim, près de Colmar, le 27 octobre 1928 (p. 233).

\section{La confiance dans le Dieu protecteur}

Né à Riquewihr le 16 février 1921, Frédéric Hunzinger a écrit 407 lettres, toutes en français, à sa future épouse, qui avait été aussi sa "Cheftaine » aux Éclaireurs unionistes de France, le mouvement scout protestant. Il signe ses lettres du nom de son totem, "girafe dévouée ». Aussi n'est-il pas surprenant que, dès sa lettre du 20 octobre 1942, après avoir mentionné les faibles quantités de nourriture distribuées aux incorporés de force, il affirme: "J'ai confiance en Dieu et il nous a toujours sauvés quand le danger était près. Je veux être son serviteur et je sais qu'il va nous rassembler tous, le jour où nous serons libérés de ces païens » (p. 23). L’affirmation, assez générale, de la foi-confiance en Dieu débouche sur une critique du paganisme nazi: les protestants alsaciens

4 Les incorporés n'avaient pas le droit de donner d'indication géographique dans leurs courriers; le 21 juin 1944, Alfred Hennes, né le 20 février 1919 à Bischheim, contourne cette interdiction en écrivant, de Roumanie, un long poème acrostiche; les premières majuscules de chaque paragraphe forment le message suivant: «Befinde mich zwieschen Jassy und Bacau [Je me trouve entre Jassy et Bacau] ». Dans ce long poème, l'auteur écrit notamment : «Plus d'un ne verra plus son Alsace » (p. 170). De fait, Alfred Hennes est décédé à Tambov, le 23 juin 1945.

5 À propos de ces soldats, voir Jean-Noël Grandhomme, Les Malgré Nous de la Kriegsmarine, Strasbourg, La Nuée Bleue, 2011. 
avaient été très bien informés du Kirchenkampf, avant d'être confrontés eux-mêmes au néo-paganisme nazi, soucieux d'éradiquer la foi chrétienne en Alsace et en Moselle ${ }^{(6)}$. Le 29 octobre 1942, Hunzinger termine sa lettre à Robert Preiss par une citation biblique, "Ist Gott mit uns, wer mag wider uns sein [Si Dieu est avec nous, qui sera contre nous?] ", que les éditeurs n'ont pas identifiée (contrairement à ce qu'ils écrivent, cette citation n'est nullement altérée) : il s'agit de l'épître de Paul aux Romains, chapitre 8, verset 31 (p. 24), et ce sont les seuls mots allemands dans une lettre rédigée en français. La piété protestante était nourrie par la lecture de la Bible de Luther, par les cantiques, par les prédications ou - pour les plus jeunes - le catéchisme, tous en langue allemande dans les paroisses luthériennes durant l'entre-deux-guerres ${ }^{(7)}$.

Jacques Baltzer, né le 2 septembre 1915 à Imbsheim, près de Saverne, est un père de famille qui écrit une lettre par jour à sa "chère douce petite femme ". Lui aussi exprime, dix jours avant sa disparition et même s'il craint de ne plus voir la fin de la guerre, sa confiance en Dieu: "J'ai toujours confiance en Dieu, qu'il vous garde tous en bonne santé et qu'il me ramène, moi aussi, auprès de vous » (p. 84, 18 octobre 1943). De manière un peu naïve, Charles Kehres, né le 11 août 1924 à Gambsheim, écrit à son amie Thérèse, quelques semaines avant de décéder en Russie: « Toujours bon courage, ne pas oublier la prière et Dieu va nous protéger » (p. 158, 31 mai 1944). Les propos de Paul Bernhardt, né le 10 novembre 1911, traduisent une piété plus mûre; à la fin de sa lettre, adressée de Pologne, il se demande si la joie de recevoir des lettres sera aussi ôtée aux soldats: "Mais ce souci, nous le laissons au grand Créateur et soutien [Helfer]. Il sait ce dont nous avons besoin et quel fardeau nous pouvons supporter. À l'instant, je me souviens du cantique [Lied ${ }^{(8)}$ " "Ce que Jésus prend en main [Was Jesus in die Hände nimmt]", où il est dit si joliment à la fin: "Comme il le fait, c'est magnifique, cela me sera salutaire [Wie er es macht ist's herrlich, wirds heilsam mir sein]." Nous allons également suivre cela, car cette épreuve doit être salutaire ${ }^{(9)}$ pour tous les gens » (p. 193, 9 août $1944^{(10)}$ ). Quelques mois auparavant, Paul Bernhardt avait écrit à son épouse, qui attendait son troisième enfant: «Tu ne dois pas craindre les heures difficiles qui vont arriver pour toi, car notre Sauveur au ciel connaît nos petits et nos grands soucis [voir Matthieu 6, v. 25-34], et il sera à tes côtés, si nous le prions avec ardeur. Car le vrai secours et l'aide viennent de lui, lui seul peut nous donner la force pour voir l'avenir avec calme. Nous devons seulement lui faire confiance et croire en lui. » Quant à lui, il estime avoir « tout ce qu'on peut souhaiter sur la terre en tant que

6 Voir Didier Sturtzer, Les Églises protestantes d'Alsace durant la seconde guerre mondiale, mémoire de maîtrise, Faculté de Théologie protestante, Université des Sciences humaines de Strasbourg, 1983.

7 Voir Catherine Storne-Sengel, Les Protestants d'Alsace-Lorraine de 1919 à 1939: entre les deux règnes, Publications de la Société savante d'Alsace, 2003.

8 Les éditeurs ont traduit malencontreusement par « chanson».

9 Les éditeurs ont traduit: « doit être le salut ».

10 Ce cantique n'est guère connu dans la tradition luthérienne. Nous avons consulté plusieurs spécialistes des cantiques protestants, en Alsace notamment (ainsi, Annemarie et Marc Lienhard), mais ils n'ont pas pu nous renseigner sur l'auteur de ce cantique ni sur la date et le lieu de sa première parution. Des recherches sur Internet menées par Yves Keler et par Marc Hug ont permis de trouver seulement l'extrait suivant: «Was Jesus in die Hände nimmt,/ und sei es noch so klein,/ das muss, ich weiss es ganz bestimmt,/ mir auch zum Segen sein. » Elles ont mis aussi en évidence que, actuellement, le cantique fait partie d'un volume intitulé Glaubenslieder, dont une $10^{\mathrm{e}}$ édition est parue en 2006 à Dillenburg (Hesse). 
mari et père d'une famille heureuse et en bonne santé », bonheur qu'il a trouvé " avec l'aide de Dieu » (p. 154, 21 mai 1944).

Le 12 mai 1944, André Frey, né le 9 septembre 1919 à Scherwiller, dans la région de Sélestat, écrit à sa mère et à sa famille. Même si la Fête des mères a été instituée par Hitler, avec d'autres incorporés de force il adopte cette tradition: « [...] la Fête des mères, et en plus au joli mois de mai, nous ne pouvons pas la passer sous silence. [...] Je lui [= maman] souhaite pour l'avenir bonne santé et bonne humeur, que le Seigneur la protège et nous la garde en vie encore de nombreuses années. [...] Et si Dieu veut que Bernard et moi rentrions, elle ne doit recevoir que du bonheur de notre part» (p. 144).

\section{La pratique religieuse}

Les lettres des Malgré Nous témoignent aussi de la distance que les incorporés peuvent prendre avec les cultes célébrés au front, dans la mesure où ils y perçoivent un mélange de religion et de patriotisme nazi. Ainsi, le 25 juillet 1943, le catholique Alphonse Lazarus, qui disparaîtra quelques mois plus tard en Ukraine, écrit-il: «Ce matin, nous avions de nouveau un service religieux de campagne [Feldgottesdienst] vers six heures moins le quart, il y avait une sainte messe et un sermon, mais il ne nous a pas du tout intéressé, car c'est comme partout: la patrie avant tout. Quand on voit l'autel, dont les pieds sont entourés du drapeau à croix gammée... Mais ça n’a pas trop d'importance, nous avons de nouveau reçu les saints sacrements et participé à une messe, ce qui est le plus important » (p. 65). Un protestant eût, probablement, parlé du contenu du sermon.

D’autres perdent les repères de l'année ecclésiastique: "Nous ne savions même pas qu'aujourd'hui, c'était Jeudi saint, mais comme les cloches se sont mises à sonner, j'ai pensé pour moi à la maison. » Or, passer le temps des fêtes loin du foyer s'avère particulièrement douloureux: "Vous pouvez vous imaginer que cela m'a fait mal lorsque j'ai entendu ça, mais je ne veux pas vous écrire plus à ce sujet. J'espère que nous fêterons Pâques à la maison l'année prochaine » (p. 126; lettre de Lucien Pfeil, né le 10 décembre 1925 à Kriegsheim, 5 avril 1944). De son côté, Jean Schaeffer, né le 27 octobre 1919 à Melsheim, évoque, quelques semaines auparavant et avec une ironie grinçante, « la charmante mélodie des obus » qu'ils entendront à nouveau chanter « quand les cloches de Pâques résonneront au pays » (p. 110). Pour Roger Berton, qui se trouve en Lettonie, « du dimanche on ne remarque pas de différence avec un autre jour, l'ennemi en face a chanté presque tout le matin depuis 7 heures " (p. 209, 3 septembre 1944; B. est né le 23 février 1912 à Lutzelhouse). Sa lettre traduit l'expérience selon laquelle « Not lehrt beten [la détresse apprend à prier] »: "Inutile de vous dire que je prie souvent et qu'ici, on apprend bien à prier, surtout dans les moments durs » (ibid.).

Georges Bordmann, né le 20 février 1909 à Niederentzen, se rend à l'église de Kassel un peu plus de deux mois après son incorporation: "Il n'y avait pas autant de fidèles qu'à Lingen. Le prêtre a annoncé une messe de requiem pour un père de famille qui est mort dans un hôpital militaire en France, et pour son fils tombé sur le front de l'Est » (p. 188, 6 août 1944).

Le Mosellan Aloïs Tempel, né le 27 juin 1921 à Niderviller, dans une région catholique très pratiquante, ne se contente pas d'évoquer sa propre pratique; il encourage aussi vivement son épouse à se rendre à l'église: «Je viens de rentrer de l'église. Ce matin, 
nous sommes pour la première fois un peu tranquilles. Sûrement que tu étais aussi à la messe, je l'espère au moins. Chérie, va le dimanche à la messe; fais aussi ta Pâques, aussi si je ne suis pas là. Fais ça pour moi, chérie; ça m’a fait mal quand je t'entendais quelques fois parler si méchamment. Pourtant, tu n'es pas mauvaise. Je ne suis pas le plus gentil, tu le sais bien. Il fait bon au lit le dimanche matin, ça sera un petit sacrifice, mais tu [ne ?] le fais pas pour le curé, tu le fais pour moi » (p. 116, lettre du 20 mars 1944).

Plus tragiquement, d'autres Malgré Nous évoquent des rites liés au décès de leurs camarades: écrivant à la famille de Jules Fuchs, décédé le 9 septembre 1944, Marcel Thomas la réconforte par le fait que son camarade a une sépulture; de plus, « devant sa tombe ouverte, je récitais un Ave Maria à votre place" (p. 226, 24 octobre 1944).

Seuls deux courriers de femmes sont publiés dans ce recueil. L'un d'eux est une brève carte postale $^{(11)}$. Par contre, la lettre de Marguerite Karlen, née le 23 mai 1926 à Bollwiller, est pleine d'intérêt. Affectée à une batterie de la défense anti-aérienne au sudouest de Leipzig, en Saxe-Anhalt, la jeune fille dépeint des lieux qui lui sont étrangers: " J'ai l'impression d'être dans une région comme en Pologne. Les gens, les maisons, la boue et même les noms de villages, Uistritz et Markrölitz, etc. Ici, les femmes portent toutes des bottes, ont un fichu sur la tête, les hommes avec leurs voitures tombereau et leurs chars à banc, dans la fange et la boue, tel que dans le film Heimkehr [1941] » (p. 228). Ces observations s'étendent aussi à la religion: "Les gens ici sont très différents du Westerwald ${ }^{(12)}$. Ils sont protestants et croient au Führer. Les églises sont murées, tout à l'air triste » (ibid.).

\section{Des lettres adressées à des ecclésiastiques}

S’il n'existe pas de lettre d'ecclésiastique parmi les témoignages retenus, on relèvera, par contre, deux courriers adressés par des soldats à leur curé.

Alors que, en décembre 1942, Antoine Berring avait été incorporé dans la Wehrmacht sur le front Est, c'est depuis les Vosges, « entre Saint-Dié et Rambervilliers » que le jeune homme, né le 28 novembre 1924 à Flexbourg, écrit, "sur une caisse de munitions ", à son "lieber Herr Pfarrer». Ce courrier du 15 octobre 1944 s'inscrit dans une série d'échanges épistolaires, puisque Antoine Berring remercie son curé « de tout cœur » pour sa lettre du 6 septembre. Ses propos ressortissent au genre de la confession, car il ne cache pas à son curé les pensées religieuses contradictoires qui surgissent au plus fort des combats: «Pour le moment, c'est tranquille. Mais, ô Jésus, il y a près de dix jours et dimanche dernier, nous étions tous moralement à bout [moralisch fertig] dans nos trous à ciel ouvert, au milieu des cris des blessés. Monsieur le Curé, vous pouvez me croire (ou pas, et je ne voudrais nullement faire le fanfaron), je n’ai jamais vécu en Russie quelque chose comme l'efficacité (sur le moral) des mortiers. Tout comme, de rage, on blasphème volontairement ou involontairement, on en vient aussi à penser, dans la plus grande détresse ou plutôt, dans une crainte mortelle [Todesangst] -, au divin [das überirdische], à notre Seigneur Dieu et à tous les êtres chers qui se trouvent à la maison » (p. 222; traduction modifiée par nos soins sur la base de l'original, p. 223). Ainsi, la détresse n'apprend-elle pas seulement à prier; elle suscite aussi le blasphème. Par contre, nulle haine, dans les

11 Madeleine Pflumio (née le 21 juillet 1926 à Strasbourg; carte du 2 octobre 1944, p. 220).

12 Entre juin et octobre 1944, Marguerite Karlen s'était trouvée au camp de Stahlhofen, dans le Westerwald. 
propos du jeune " paroissien », ainsi qu'il signe sa lettre, à l'endroit des troupes adverses: « Nous sommes face à beaucoup d'Américains qui ne sont pas pires que d'autres. Ce ne sont pas du tout des gangsters comme on nous les présentait» (ibid.). Antoine Berring mourut une semaine plus tard, à Brouvelieures, dans les Vosges.

La missive qu'Alfred Haenel, âgé d'à peine dix-huit ans, envoie le 29 avril 1944 depuis Feltin (Estonie) à ses « chers camarades de classe " et à son " cher Monsieur le Curé », à Schirrhein, est plus tragique encore: le même jour, le jeune homme va être fusillé pour avoir voulu fuir devant l'ennemi russe. «Des circonstances particulières ont voulu que je doive subir la mort. Je conçois que, dans les circonstances actuelles, des lois sévères soient en vigueur, et la roue passe aussi sur moi » (p. 253, traduction légèrement modifiée). À ses camarades de classe, le natif de Bischwiller adresse ses chaleureuses salutations et les prie de le garder en bon souvenir; à son curé, il demande de se souvenir de lui devant l'autel et de transmettre ses salutations à ses amies et à ses camarades. À tous, il demande d'adresser ses salutations à la chorale - sans doute le chœur paroissial, dont il faisait partie ${ }^{(13)}$.

\section{Conclusion}

En raison du petit nombre de lettres (moins de 120) que renferme ce volume, les conclusions qui suivent devront être considérées avec prudence: en effet, nous ignorons si ces lettres, dont les éditeurs ne livrent pas les « critères précis » (p. 12) de sélection (de fait, ils ont privilégié un certain nombre de séries ${ }^{(14)}$ ), sont représentatives de la masse des 5000 lettres conservées par la Société de l'Histoire de la Poste.

Parmi les images de Dieu que privilégient les Malgré Nous, le Dieu protecteur ${ }^{(15)}$ ou la providence ${ }^{(16)}$ - vient en première place, ce qui n'a rien de surprenant. C'est la même image de Dieu qui domine dans les lettres envoyées par les Jésuites allemands qui servirent dans la Wehrmacht ${ }^{(17)}$, et, plus largement, dans la correspondance des

13 Johannes GüsGEN, «Die Bedeutung der Katholischen Militärseelsorge in Deutschland von 1933-1945 », in: Müller/Volkmann (éd.), Die Wehrmacht (note 2), p. 503-524, ne parle malheureusement pas de la cure d'âme auprès des soldats condamnés à mort; par contre D. BEESE, "Kirche im Krieg » (note 2), p. 497-502, évoque longuement le sort des soldats, souvent très jeunes, condamnés à mort pour avoir fui le combat devant l'ennemi, ou, notamment, pour s'être endormis à la suite de marches harassantes, et alors qu'ils devaient monter la garde. Ces cas tragiques provoquèrent souvent, chez les aumôniers protestants qu'il a interviewés, de difficiles problèmes de conscience; les ecclésiastiques étaient tenus d'accompagner les soldats par leur cure d'âme depuis la découverte du « délit » jusqu'à l'exécution de la sentence.

14 Telle a aussi été la démarche adoptée par Klaus Latzel, Deutsche Soldaten - nationalsozialistischer Krieg? Kriegserlebnis - Kriegserfahrung 1939-1945, Paderborn/Munich/Vienne/Zurich, Schöningh, 1998, qui a étudié, pour la Seconde Guerre mondiale, 2749 lettres écrites par 22 personnes (p. 107).

15 Outre les références mentionnées, voir la lettre du 19 mars 1944 de Désiré Eiller, né le 10 mai 1916 à Haguenau: «Tu sais, [...] la pensée d'être loin de vous me met en boule, mais comme dit, j'ai confiance en Dieu, qu'il nous protègera et mettra une fin bientôt à toute cette horreur et que nous puissions nous revoir bientôt » (p. 115).

16 «Ce qui nous attend encore, nous qui avons été épargnés par le destin, repose entre les mains de Dieu» (p. 147, Jean Schaeffer, le 12 mai 1944). Le même soldat écrit: " Nous sommes malheureusement nés à une mauvaise époque. »

17 Antonia Legers, Jesuiten in der Wehrmacht. Kriegslegitimation und Kriegserfahrung, Paderborn/ Munich/Vienne/Zurich, Schöningh, 2009, p. 99; les soldats parlent notamment de «Vorsehung» et de "Führung". 
soldats allemands ${ }^{(18)}$. Toutefois, dans cette dernière correspondance comme chez les Malgré Nous, nombreux sont les soldats qui ne disent mot de Dieu, pas même du secours providentiel. Klaus Latzel a mis en évidence que leur proportion était bien plus forte (14 sur 22, soit près de deux tiers) que durant la Première Guerre mondiale (5 sur 17 , soit moins d'un tiers) ${ }^{(19)}$; cette évolution pourrait témoigner des succès de la propagande athée des nazis (et de la "religion politique » centrée sur le Führer), mais sans doute exprime-t-elle aussi, plus largement, la progression de la sécularisation en Occident dans la première moitié du $\mathrm{xx}^{\mathrm{e}}$ siècle. Il conviendrait d'examiner si cette évolution s'est dessinée aussi en Alsace et en Moselle. On relèvera en tout cas que, dans la correspondance des Malgré Nous, les demandes de prier pour soi sont peu nombreuses (voir p. 206, lettre du 27 août 1944, Roger Berton).

Les soldats alsaciens et mosellans évoquent la "volonté de Dieu [Gottes Wille] »: «Si seulement, par la volonté de Dieu, cette guerre se terminait enfin!» (p. 217; Marcel Thomas, 18 septembre 1944). Toutefois, au contraire des soldats allemands, jamais on ne trouve sous leur plume l'idée que la guerre menée contre l'Union soviétique serait le juste jugement de Dieu pour punir le bolchevisme athée, et les soldats de la Wehrmacht les instruments de la vengeance divine. Il est vrai que les Malgré Nous ont été mobilisés en 1942, et que ce thème du jugement domine surtout en 1941, au moment de l'offensive éclair contre l'Union soviétique ${ }^{(20)}$.

Alors que, dans les correspondances de la Première Guerre mondiale, on trouve souvent des interrogations sur la théodicée (si Dieu est bon, pourquoi laisse-t-il faire cela? ou alors, n'est-il pas tout puissant?) ${ }^{(21)}$, ces douloureux questionnements sont absents des lettres ici rassemblées. S'agit-il d'un " effet de source", compte tenu de la faible proportion de lettres de Malgré Nous éditées? En tout cas, ce thème apparait chez d'autres soldats de la Wehrmacht ${ }^{(22)}$. Quant à la présence du curé - et, par contraste, l'absence du pasteur -, dit-elle quelque chose d'un lien moins fort entre le chargé d'âmes protestant et ses ouailles? Sur ce point aussi, il est prudent de ne pas tirer de conclusion hâtive.

Bien entendu, ces lettres, extrêmement riches, pourraient faire l'objet d'autres investigations. Elles témoignent, par exemple, de l'impréparation de l'armée allemande lors de l'offensive en Russie: « Chère maman, envoie-moi des gants et un passe-montagne, écrit Pierre Constans, né le 25 novembre 1920, sinon mes mains vont geler, puisque nous n'avons pas encore nos effets d'hiver et qui sait s'il y en aura » (p. 83, 15 octobre 1943). D’autres soldats évoquent l'arrestation des Juifs; c'est le cas de Jean-Pierre Bader, qui écrit depuis Arta, dans le nord de la Grèce: « Un soir, nous eûmes l'ordre d'arrêter tous les Juifs d'Arta. Ce fut une razzia, des hurlements et des cris des femmes. Nous avons livré ces Juifs aux Grecs, je ne sais pas ce qu'ils en ont fait. Ce fut une action générale dans toute la Grèce et dans tout le pays, le peuple de Dieu fut arrêté » (p. 136,

18 Voir K. Latzel, Deutsche Soldaten (note 14), p. 294. Les verbes qui sont associés à Dieu sont beistehen, beschützen ou encore helfen.

19 Ibid., p. 295. Voir aussi p. 268 sq.

20 Voir A. Legers, Jesuiten in der Wehrmacht (note 17).

21 Ibid., p. 93-96.

22 Ibid., p. 106 sq. 
20 avril 1944). Il serait intéressant de consacrer, pour l'ensemble de la correspondance des Malgré Nous, une étude spécifique à ce thème ${ }^{(23)}$.

Toutefois, les pages qui précèdent montrent l'importance de la religion dans la correspondance des Malgré Nous, et les commentaires que nous donnons tout l'intérêt qu'il y aurait eu à associer des théologiens à la publication de ces lettres, si riches et si émouvantes.

\section{Résumé}

La correspondance des "Malgré Nous" (1942-1945) témoigne des préoccupations religieuses des incorporés de force alsaciens et mosellans. Leurs lettres insistent sur le Dieu protecteur, auquel ils encouragent leurs proches à prier. Elles témoignent de leur pratique religieuse, marquée par la défiance pour les cultes patriotiques nazis. Enfin, les lettres adressées à des ecclésiastiques ont valeur de confession, surtout lorsque des condamnés à mort adressent leur dernière lettre à leur curé.

\section{Zusammenfassung}

Die Korrespondenz der "Malgré Nous“ (1942-1945) bekundet die religiösen Besorgnisse der Zwangseingezogenen aus dem Elsass und aus Lothringen. In ihren Briefen insistieren sie auf dem schützenden Gott und ermutigen ihre Familie und Freunde zu ihm zu beten. Gleichzeitig lassen sie ihre religiösen Praktiken erkennen, die sich durch ein Misstrauen gegenüber den patriotischen Gottesdiensten der Nationalsozialisten auszeichnen. Schließlich können die an die Geistlichen gesendeten Briefe als Beichte gelesen werden, vor allem wenn sich die zum Tode verurteilten Männer in ihrem letzten Brief an ihren katholischen Pfarrer wenden.

23 Pourl'ensemble dela Wehrmacht, voir Walter Manoscheк (éd.), "Es gibtnureinesfürdasJudentum: Vernichtung”. DasJudenbild in deutschen Soldatenbriefen 1939-1944, Hambourg, Hamburger Edition, 1995. 